\title{
Microlensing Events Near-Field Surveys
}

\author{
Cheongho Han ${ }^{* \dagger}$ \\ Department of Physics, \\ Chungbuk National University, Chongju 361-763, Korea; \\ E-mail: cheonghodastroph.chungbuk.ac.kr
}

\begin{abstract}
We estimate the rate of near-field microlensing events expected from all-sky surveys and investigate the properties of the events. Under the assumption that lenses are composed of stars, our estimation of the event rate ranges from $\Gamma_{\text {tot }} \sim 0.1 \mathrm{yr}^{-1}$ for a survey with a magnitude limit of $V_{\text {lim }}=12$ to $\Gamma_{\text {tot }} \sim 23 \mathrm{yr}^{-1}$ for a survey with $V_{\text {lim }}=18$. We find that the average distances to the source star and lens vary considerably depending on the magnitude limit, while the dependencies of the event time scale and lens-source transverse speed are weak. We also find that the the average lens-source proper motion of events expected from a survey with $V_{\text {lim }}=18$ would

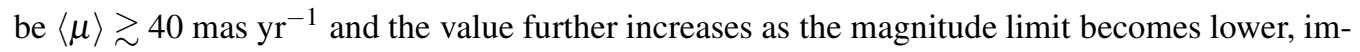
plying that the source and lens of a significant fraction of near-field events can be resolved from high-resolution follow-up observations conducted several years after the lensing magnification.
\end{abstract}

The Manchester Microlensing Conference: The 12th International Conference and ANGLES Microlensing Workshop

January 21-25 2008

Manchester, UK

\footnotetext{
${ }^{*}$ Speaker.

${ }^{\dagger}$ We thanks for the support from Chungbuk National University Grant.
} 


\section{Introduction}

Recently, a detection of a lensing event occurred on a nearby star was reported by [1] and [2]. The lensed star (GSC 3656-1328) is located about $1 \mathrm{kpc}$ from the sun in the disk of the Milky Way. This detection along with the advent of transient surveys capable of covering a very wide field with high cadence such as ASAS [3], ROTSE [4], TAROT and ARAGO [5], and Pan-Starrs [6] have drawn attention of many researchers in microlensing community on the feasibility of near-field microlensing surveys. If near-field events could be detected from such surveys, they would be able to provide precious information about the matter distribution around the sun including dark objects. In addition, these surveys might enable detections of close planets with the microlensing technique that has demonstrated its capability in detecting distant planets.

In this paper, we estimate the rate of lensing events in terms of event per year and to obtain the distributions of the physical parameters and observables of events expected from all-sky lensing surveys with various magnitude limits.

\section{Lensing Simulation}

To investigate the rate and properties of near-field lensing events, we conduct Bayesian simulation of these events. The basic scheme of the simulation is as follows.

1. We first produce source stars on the sky that can be seen from a survey with a given magnitude limit. We assign the locations on the sky and the distances to the stars based on a spatial mass distribution model of the Galaxy. The stellar brightness is assigned based on a model luminosity function considering distances to stars and extinction.

2. Once source stars are produced, we then produce lenses. We assign the lens masses based on a model mass function. The distances to the lenses are allocated from the same spatial mass distribution model as that of the source star.

3. The lens-source transverse speed of each event is computed from the velocities of the lens and source, which are assigned based on a dynamic model of the galactic disk.

4. With all the lens parameters, we then produce events and estimate the event rate by weighting the individual events with an appropriate lensing probability.

\section{Results}

In Table 1, we list the total event rate, $\Gamma_{\text {tot }}$, expected from surveys with various magnitude limits. Also listed in the table are the total number of stars that can be monitored from the surveys, $N_{\star, \text { tot }}$, and the average optical depth to lensing, $\langle\tau\rangle$. We find that our estimation of the optical depth well matches the estimation of [7]. The multiplication $N_{\star, \text { tot }} \times\langle\tau\rangle$ represents the number of stars undergoing lensing magnification at a given moment, which was presented in [7]. We also find a good match between his and our estimations. The estimated event rate ranges from $\Gamma_{\text {tot }} \sim 0.1$ per year for a survey with a magnitude limit of $V_{\lim }=12$ to $\Gamma_{\text {tot }} \sim 23$ per year for a survey with 


\begin{tabular}{cccc}
\hline \hline$V_{\lim }$ & $N_{\star \text { tot }}$ & $\langle\tau\rangle$ & $\Gamma_{\text {tot }}\left(\mathrm{yr}^{-1}\right.$ \\
\hline 12 & $0.10 \times 10^{7}$ & $0.12 \times 10^{-8}$ & 0.08 \\
14 & $0.47 \times 10^{7}$ & $0.25 \times 10^{-8}$ & 0.57 \\
16 & $2.0 \times 10^{7}$ & $0.52 \times 10^{-8}$ & 3.8 \\
18 & $7.5 \times 10^{7}$ & $0.92 \times 10^{-8}$ & 23.0 \\
\hline
\end{tabular}

Table 1: Rates of near-field microlensing events, $\Gamma_{\text {tot }}$, expected from all-sky surveys with various magnitude limits, $V_{\text {lim. }}$. Also listed are the total number of stars that can be monitored from the surveys, $N_{\star, \text { tot }}$, and the average optical depth to lensing, $\langle\tau\rangle$.

$V_{\lim }=18$, confirming the result of [7] that the lensing probability rapidly increases with the increase of the magnitude limit. Two factors contribute to the increase of the event rate with the increase of the magnitude limit. The first factor is the increase of the number of source stars and the other factor is the extension of the line of sight toward source stars and thus increase of the optical depth. We find that the former factor is more important.

In Figure 1, we present the distributions of lens parameters and the observables of events including the distributions of the source star brightness, distances to source stars and lenses, lens masses, lens-source transverse speed, event time scale, and lens-source relative proper motion. The event time scale is determined by the Einstein time scale, which is required for the source to transit the Einstein radius of the lens, i.e. $t_{\mathrm{E}}=r_{\mathrm{E}} / v$. The proper motion corresponds to $\mu=\theta_{\mathrm{E}} / t_{\mathrm{E}}$, where $\theta_{\mathrm{E}}=r_{\mathrm{E}} / D_{\mathrm{L}}$ is the angular Einstein radius. In Table 3, we also present the average values of the lens parameters. From Figure 1 and Table 1 and 2 we find the following tendencies.

1. The distributions of $D_{\mathrm{S}}$ and $D_{\mathrm{L}}$ vary considerably depending on the magnitude limit. The trend, as expected, is that the distances to the source and lens become larger as fainter stars are monitored.

2. On the other hand, the dependency of the distribution of the lens mass, event time scale and transverse speed on the magnitude limit are weak. The mean value of the lens mass is $\langle M\rangle \sim 0.2 M_{\odot}$, implying that the majority of events will be caused by low-mass stars.

3. With the increase of the magnitude limit, the distance to the lens increases while the transverse speed remains nearly the same. As a result, the average proper motion $\mu=\theta_{\mathrm{E}} / t_{\mathrm{E}}=$ $v_{\mathrm{t}} / D_{\mathrm{L}}$ of events decreases as the magnitude limit increases. However, we note that even for

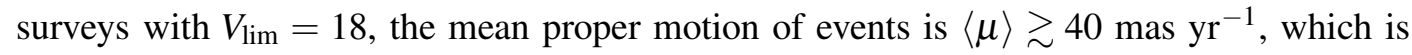

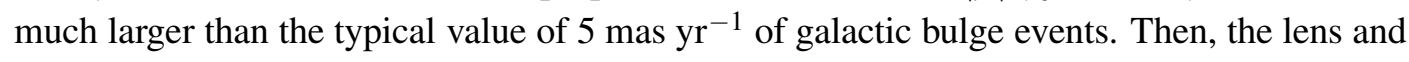
source of a significant fraction of near-field events can be resolved from follow-up observations by using high-resolution instrument such as the Hubble Space Telescope conducted several years after the peak of the magnification. Resolving of the lens and source not only enables the measurement of the proper motion but also helps to identify the lens.

\section{Conclusion}

Instigated by the recent discovery of an event occurred on a nearby star, we estimated the rate 

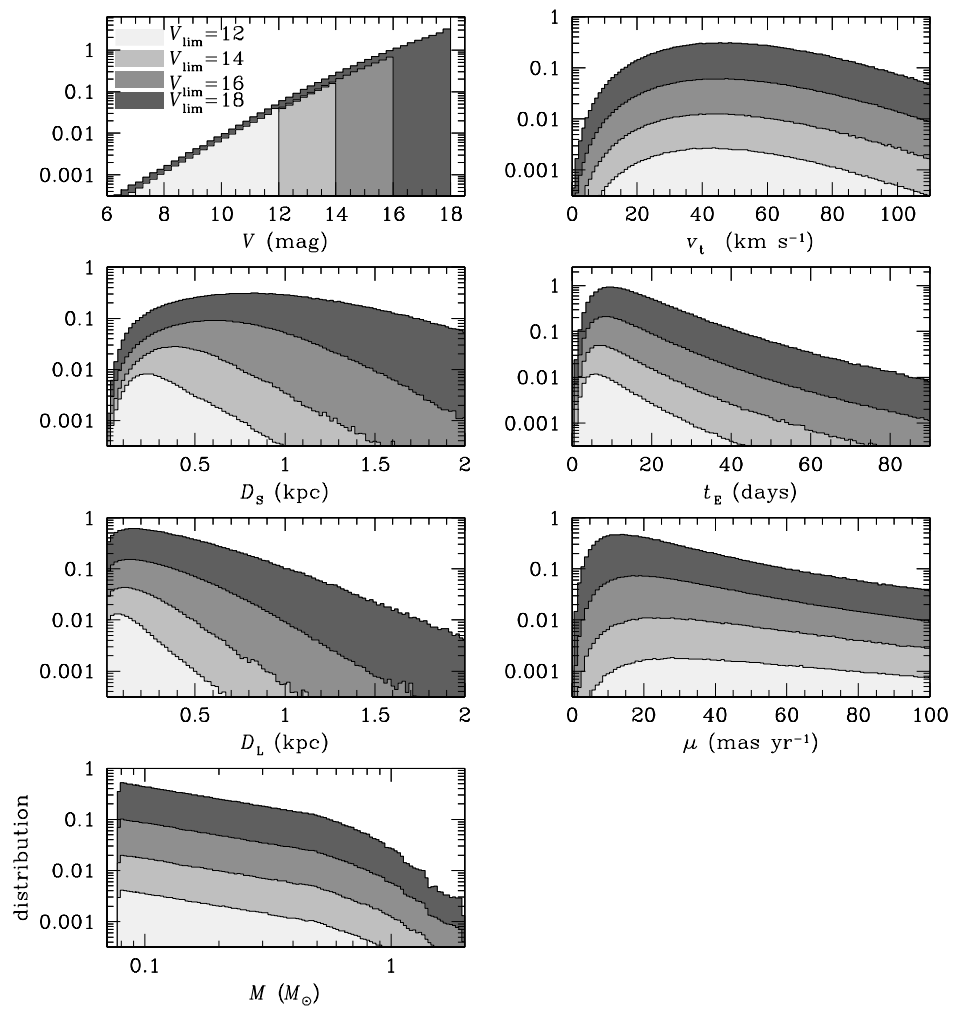

Figure 1: Distributions of lens parameters and observables of near-filed microlensing events expected from all-sky surveys with various magnitude limits, $V_{\mathrm{lim}}$. From the left top, the individual panels represent the distributions of the source star brightness $(V)$, distances to the source star $\left(D_{\mathrm{S}}\right)$ and lens $\left(D_{\mathrm{L}}\right)$, lens masses, transverse speeds $\left(v_{\mathrm{t}}\right)$, event time scales $\left(t_{\mathrm{E}}\right)$, and lens-source relative proper motions $(\mu)$, respectively. The mean values of the parameters and observables are listed in Table 2.

\begin{tabular}{ccccccc}
\hline \hline $\begin{array}{c}V_{\lim } \\
(\mathrm{mag})\end{array}$ & $\begin{array}{c}\left\langle D_{\mathrm{S}}\right\rangle \\
(\mathrm{kpc})\end{array}$ & $\begin{array}{ccccc}\left\langle D_{\mathrm{L}}\right\rangle \\
(\mathrm{kpc})\end{array}$ & $\begin{array}{c}\langle M\rangle \\
\left(M_{\odot}\right)\end{array}$ & $\begin{array}{c}\left\langle v_{\mathrm{t}}\right\rangle \\
\left(\mathrm{km} \mathrm{s}^{-1}\right)\end{array}$ & $\begin{array}{c}\left\langle t_{\mathrm{E}}\right\rangle \\
(\text { days })\end{array}$ & $\begin{array}{c}\langle\mu\rangle \\
\left(\mathrm{mas} \mathrm{yr}^{-1}\right)\end{array}$ \\
\hline 12 & 0.36 & 0.18 & 0.18 & 54.7 & 13.4 & 73.7 \\
14 & 0.49 & 0.25 & 0.18 & 55.5 & 15.4 & 60.7 \\
16 & 0.72 & 0.35 & 0.18 & 56.6 & 17.4 & 49.3 \\
18 & 1.03 & 0.46 & 0.18 & 57.4 & 19.9 & 41.7 \\
\hline
\end{tabular}

Table 2: Average values of the lens parameters and observables of near-field microlensing events expected from wide-field surveys with various magnitude limits, $V_{\text {lim }}$. 
of near-field lensing events expected from all-sky surveys with various magnitude limits. Under the assumption that all lenses are composed of stars, our estimation of the event rate ranges from $\Gamma_{\text {tot }} \sim 0.1$ per year for a survey with a magnitude limit of $V_{\lim }=12$ to $\Gamma_{\text {tot }} \sim 23$ per year for a survey with $V_{\lim }=18$, confirming the previous result that lensing probability rapidly increases with the increase of the magnitude limit. The increase of the rate is due to two factors which are the extension of the line of the sight toward source stars and the increase of the number of source stars. We found that the latter factor is more important. We also investigated the distributions of the physical parameters of lens systems and the observables of these events. From this, we found that although the average distances to source stars and lenses vary considerably depending on the magnitude limit, the dependencies of the the lens mass, event time scale, and lens-source transverse speed are weak. We also found that the the average lens-source proper motion of events expected

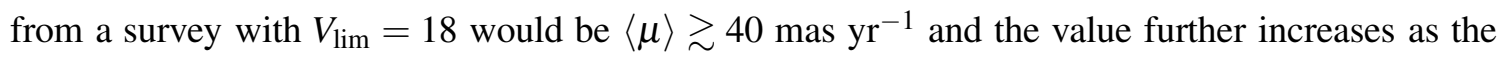
magnitude limit becomes lower. This implies that the source and lens of a typical near-field lensing event can be resolved from high-resolution follow-up observations conducted several years after the peak of the lensing magnification.

This work was supported by the basic research fund from Chungbuk National University.

\section{References}

[1] Gaudi, B. S., et al. 2007, ApJ, submitted

[2] Fukui, A., et al. 2007, preprint (arXiv:0708.1066v1)

[3] Szczygieł, D. M., \& Fabrycky, D. C. 2007, MNRAS, 377, 1263

[4] Akerlof, C. W., et al. 2003, PASP, 115, 132

[5] Boër, M. 2001, Astronomische Nachrichten, 322, 343

[6] Hodapp, K. W., et al. 2004, Astronomische Nachrichten, 325, 636

[7] Nemiroff, R. J. 1998, ApJ, 509, 39 